\title{
Strategies for high-resolution imaging of radiation-sensitive materials in an aberration-corrected transmission electron microscope
}

Ute A. Kaiser

Ulm University, Group of Electron Microscopy of Materials Science, Ulm, Germany.

The practical realisation of aberration correction enabled low-voltage and high-resolution transmission electron microscopy which is relevant for structural, chemical and electronic analysis of such electron radiation-sensitive materials, which are suffering predominantly on displacements of atoms by elastic binary collisions between the beam electrons and the sample atoms, the so called knock-on damage. One main aim of the SALVE (sub angstrom low-voltage electron microscopy) project [1] is to explore the possibilities of high-resolution imaging of the pristine structure of a biological object and of those present and future materials, which are of low dimensionality or/and bridging the former frontier between materials and life sciences using a 20-80kV-optimised FEI microscope platform with spherical and chromatic aberration correction. In the first part of the talk we will briefly outline the current state of the SALVE microscope and show theoretical predictions for phase contrast imaging using our corrector with positive and slightly tunable fifth-order spherical aberration (C5), which is beneficial for high phase contrast if combined with a suitable small negative spherical aberration of third order (C3) and positive defocus (C1) as then the linear and non-linear contrast contributions often simply add up.

In the second part of the talk we report on structural properties of low-dimensional materials obtained at $80 \mathrm{kV}$ acceleration voltage. In particular we investigate basic crystallographic defects such as zerodimensional defect [2,3], one-dimensional defect [3,4], extended defects [3] and determine - via density functional theory calculations - their properties. We briefly present our numerical post-processing method for removing the effect of anti-symmetric residual aberrations in high-resolution transmission electron microscopy (HRTEM) images of weakly scattering 2D-objects [5].We show moreover that now even the atomic structure of an amorphous phase can be unravelled in direct space just from the analysis of high-resolution TEM images [6,7]. We demonstrate that multiple channels of damage production have to be identified as not only knock-on damage plays an important role but also damage via excitations of the electronic system of the sample, i.e., ionization or radiolysis, chemical etching by radicals produced from impurities on the sample surfaces, and heating via deposition of energy from the electron beam can be responsible if the atomic structure of a sample is degraded. As results need to be confirmed by image calculation, for high-resolution TEM images, the contribution of inelastic scattering must be taken into consideration [8]. For energy-filtered images of low-Z materials at low voltages, the contributions of elastic and inelastic scattering to the image intensity cannot be separated from each other because the inelastic scattering amplitudes are influenced by elastic scattering, and vice versa [9]. All the damage channels can be suppressed by simply limiting the total electron doses on the samples. On the other hand, limited electron doses result, however, inevitably to worse signal to noise ratios, which imposes a lower bound on the electron dose with which the features of interest in the studied sample can still be discerned. Here we show an quantitative approach for estimating the visibility of objects in TEM images with limited doses [10], to sandwich beam-sensitive objects in-between two graphene layers [11,12], and how to get graphene clean [13]. We demonstrate that lowering the energy of the electrons down to $20 \mathrm{kV}$ prevents various metal clusters and molecule inside CNTs from electronbeam-stimulated damage [14]. The exchange of the isotopes of the molecules (deuterium instead of 
hydrogen) is another strategy that enhances the stability against knock-on collisions independent on the voltage [15]. We discuss in more detail the discovery of new structures such as 2D square ice [16] and crystalline AuC [17].

In the third part we show 40kV EELS experiments for two-dimensional materials with exceptionally low background noise and explore the nature of electronic excitations by inspecting the low losses in the $0-20 \mathrm{eV}$ range. In fact, information can be obtained, under controlled illumination conditions and sample orientation, to the onset of optical transitions. In addition to the energy of electronic excitations, information on the momentum transfer can be obtained in dependence of the crystallographic direction. We determine the dispersion behaviour for $\pi$ and $\pi+\sigma$ plasmons in free-standing single-layer graphene and multilayers as benchmark experiments [18] confirming earlier calculations.

[1] U. Kaiser, J. Biskupek, J.C. Meyer, J. Leschner, L. Lechner, H. Rose, M. Stöger-Pollach, A.N. Khlobystov, P. Hartel, H. Müller, M. Haider, S. Eyhusen and G. Benner, ”Ultramicroscopy, 111 (2011) 1239 [2] J. Kotakoski, J. Meyer, S. Kurasch, D. Santos-Cottin, U. Kaiser and A. Krasheninnikov, Physical Review B, 83 (2011) 235420

[3] O. Lehtinen, H-P Komsa, A. Pulkin, M. B. Whitwick, M.-W Chen, T. Lehnert, M. Mohn, O. V. Yazyev, A. Kis, A. V. Krasheninnikov, U.Kaiser, ACSNano accepted (2015)

[4] O. Lehtinen, S. Kurasch, A.V. Krasheninnikov and U. Kaiser, Nature Communications 4 (2013) 3098

[5] O. Lehtinen, D. Geiger, Z. Lee, M. B. Whitwick, M.-W. Chen, A. Kis, and U. Kaiser Ultramicroscopy (2015) 10.1016/j.ultramic.2014.09.010

[6] P. Y. Huang, S. Kurasch, A. Srivastava, V. Skakalova, J. Kotakoski, A. V. Krasheninnikov, R. Hovden, Q. Mao, J. C. Meyer, J. Smet, D. A. Muller, and U. Kaiser Nano Lett. 12(2) (2012)1081

[7] P. Y. Huang, S. Kurasch, J.S. Alden, A. Shekhawat, A.A. Alemi, P. L. McEuen, J.P. Sethna, U. Kaiser, D.A. Muller, Science 342 (2013) 224

[8] Z. Lee, J.C. Meyer, H. Rose and U. Kaiser, Ultramicroscopy 112 (2012) 39

[9] Z. Lee, H. Rose, R. Hambach, P. Wachsmuth and U. Kaiser, Ultramicroscopy 134 (2013) 102

[10] Z. Lee, H. Rose, O. Lehtinen, J. Biskupek, U. Kaiser, Ultramicroscopy 145 (2014) 3

[11] G. Algara-Siller, S. Kurasch, M. Sedighi, O. Lehtinen and U. Kaiser, Appl. Phys. Lett. 103 (2013) 203107

[12] O. Lehtinen, I.L. Tsai, R. Jalil, R.R. Nair, J. Keinonen, U. Kaiser, I.V. Grigorieva, Nanoscale 6, (2014) 6659

[13] G. Algara-Siller, O. Lehtinen, A. Turchanin, U. Kaiser, Appl. Phys. Lett. 104 (2014) 153115

[14] T. W. Chamberlain, T. Zoberbier, J. Biskupek, A. Botos, U. Kaiser, A. N. Khlobystov, Chemical Science 3 (2012) 1919

[15] T. W. Chamberlain, J. Biskupek, S. T. Skowron, P. A. Bayliss, E. Bichoutskaia, U. Kaiser, A. N. Khlobystov, Small 11 (2014) 510

[16] G. Algara-Siller, O. Lehtinen, F.C. Wang, R. R. Nair, U. Kaiser, H. A. Wu, I. V. Grigorieva, A. K. Geim, Nature (2015) accepted

[17] B. Westenfelder, J. Biskupek, J. Meyer, S. Kurasch, X. Lin, F. Scholz, A. Groß, U. Kaiser, Scientific Reports (2015) accepted

[18] P. Wachsmuth, R. Hambach, M.K. Kinyanjui, M. Guzzo, G. Benner, U. Kaiser, Phys. Rev. B B 88 (2013) 075433

[19] Fruitful cooperation within the SALVE project and financial support by the DFG (German Research Foundation) and by the Ministry of Science, Research, and the Arts (MWK) of Baden-Württemberg are gratefully acknowledged. 